Original Research

\title{
Altered functional connectivity of primary visual cortex in adults with strabismus and amblyopia: a resting-state fMRI study
}

\author{
Li-Qi Liu ${ }^{1,2, \dagger}$, Qiu-Yu Li ${ }^{1, \dagger}$, Zhi-Hua Zhang ${ }^{1,2, \dagger}$, Shi-Nan Wu ${ }^{1}$, Hui-Ye Shu ${ }^{1}$, Li-Juan Zhang $^{1}$, Rong-Bin Liang ${ }^{1}$, Yi-Cong Pan $^{1}$, \\ YiShao ${ }^{1, *}$ \\ ${ }^{1}$ Department of Ophthalmology, The First Affiliated Hospital of Nanchang University, Jiangxi Province Clinical Ophthalmology Institute, The First \\ Affiliated Hospital of Nanchang University, 330006 Nanchang, Jiangxi, China \\ ${ }^{2}$ Department of Clinical Medicine, Queen Mary School, Nanchang University, 330031 Nanchang, Jiangxi, China \\ *Correspondence: freebee99@163.com (Yi Shao) \\ ${ }^{\dagger}$ These authors contributed equally.
}

\section{DOI:10.31083/j.jin2101004}

This is an open access article under the CC BY 4.0 license (https://creativecommons.org/licenses/by/4.0/).

Submitted: 3 August 2021 Revised: 23 November 2021 Accepted: 26 November 2021 Published: 21 January 2022

Functional connectivity of the primary visual cortex was explored with resting functional magnetic resonance imaging among adults with strabismus and amblyopia and healthy controls. We used the two-sample test and receiver operating characteristic curves to investigate the differences in mean functional connectivity values between the groups with strabismus and amblyopia and healthy controls. Compared with healthy controls, functional connectivity values in the left Brodmann areas 17, including bilateral lingual/angular gyri, were reduced in groups with strabismus and amblyopia. Moreover, functional connectivity values in the right Brodmann area 17, including left cuneus, right inferior occipital gyrus, and left inferior parietal lobule, were reduced in adults with strabismus and amblyopia. Our findings indicate that functional connectivity abnormalities exist between the primary visual cortex and other regions. This may be the basis of the pathological mechanism of visual dysfunction and stereovision disorders in adults with strabismus and amblyopia.

\section{Keywords}

Strabismus; Amblyopia; Functional connectivity; Primary visual cortex; Spontaneous activity; Resting fMRI; Neuropathology; Ophthalmopathy

\section{Introduction}

Strabismus and amblyopia (SA) are visual developmental disorders, which may occur during childhood [1]. Strabismus is the relative deviation of the visual axis, and there are a variety of methods by which it can be classified. At present, the mainstream classification method is to categorize it as esotropia, exotropia, vertical rotary strabismus, or A-V pattern strabismus, according to the oblique direction of eye movement. It can also be classified according to abnormal eye movements [2].

During the critical period of visual development, the abnormal visual experience can cause developmental diseases of the visual system, including amblyopia. Amblyopia is characterized by reduced best-corrected vision in one or both eyes, without associated ocular organic lesions [3]. A series of studies have shown that ametropia, strabismus and ambly- opia often influence each other [4-6]. Strabismus and amblyopia do not only affect visual function and appearance but can also seriously affect the psychosocial health of patients [7-9].

Strabismus is usually treated by surgery, while the removal mainly treats amblyopia of form deprivation, occlusion therapy, or depression therapy during the critical period [9]. Without treatment, SA can persist into adulthood [8]. Adults with SA may have permanent cortical changes due to disrupted functional connections in the brain, abnormal eye movements, loss of stereo vision, and interocular inhibition [10-13]. Neuroanatomically, the binoculus provides two essentially independent information streams synthesized by the primary visual cortex (V1) [14]. After fusing images through the $\mathrm{V} 1$, the visual system produces a unified perception of the visual world. Simultaneously, the stereo parallax is used to perceive the distance information of the observed object [15].

Compared with magnetic resonance imaging (MRI), functional MRI (fMRI) can detect the fluctuation of blood oxygenation in specific cerebral regions to further explore the functional state of neuronal activity in these cerebral regions. It provides new neuroimaging and brain function studies [16-18]. Furthermore, fMRI can be used to determine the pathophysiological changes of brain function under restingstate conditions, which can be helpful for clinical diagnosis and treatment evaluation.

Comparisons facilitated by dynamic fMRI showed that healthy controls (HCs) participation is simpler and easier, with fewer influencing factors and more analytic data [19]. Our paper examines the functional connectivity (FC) of the $\mathrm{V} 1$ in adults with SA using resting fMRI. Our findings may be useful in determining the cause of visual impairment and visual fusion disorders among adults $(>18+$ years of age) with SA. 


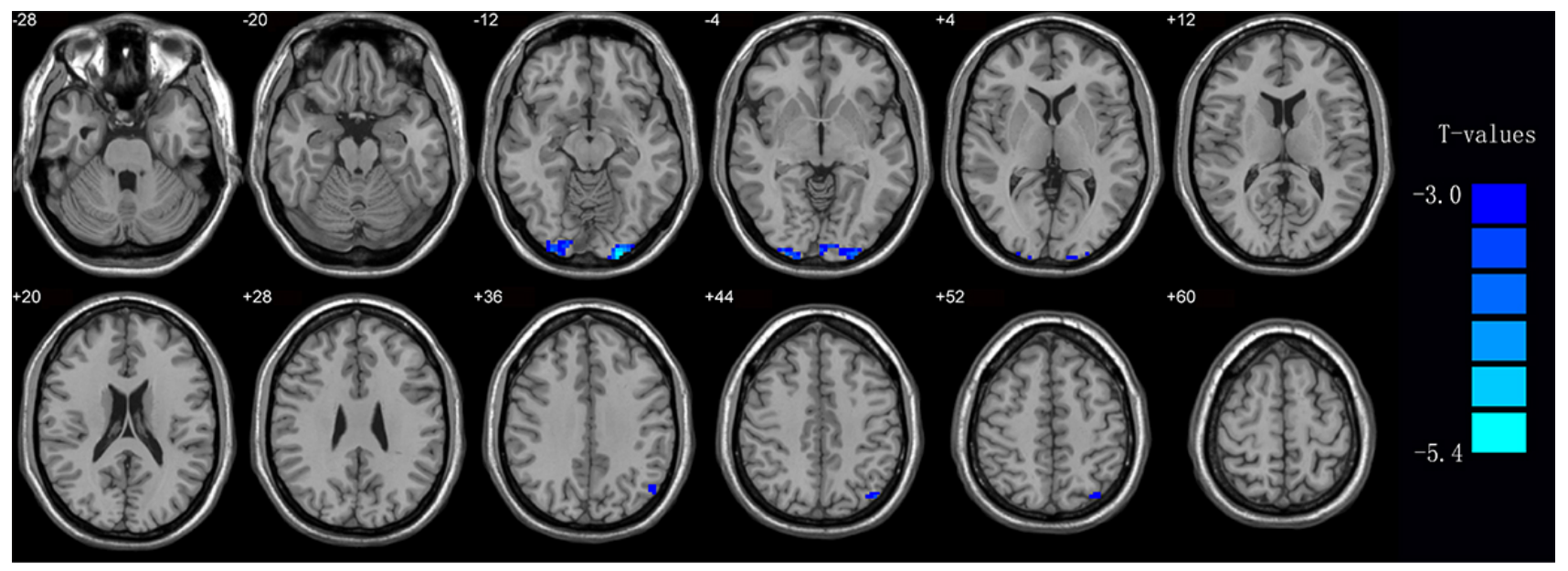

L
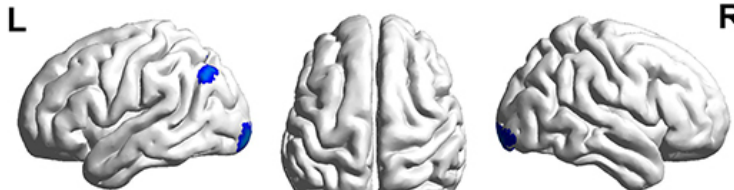

$\mathbf{R}$
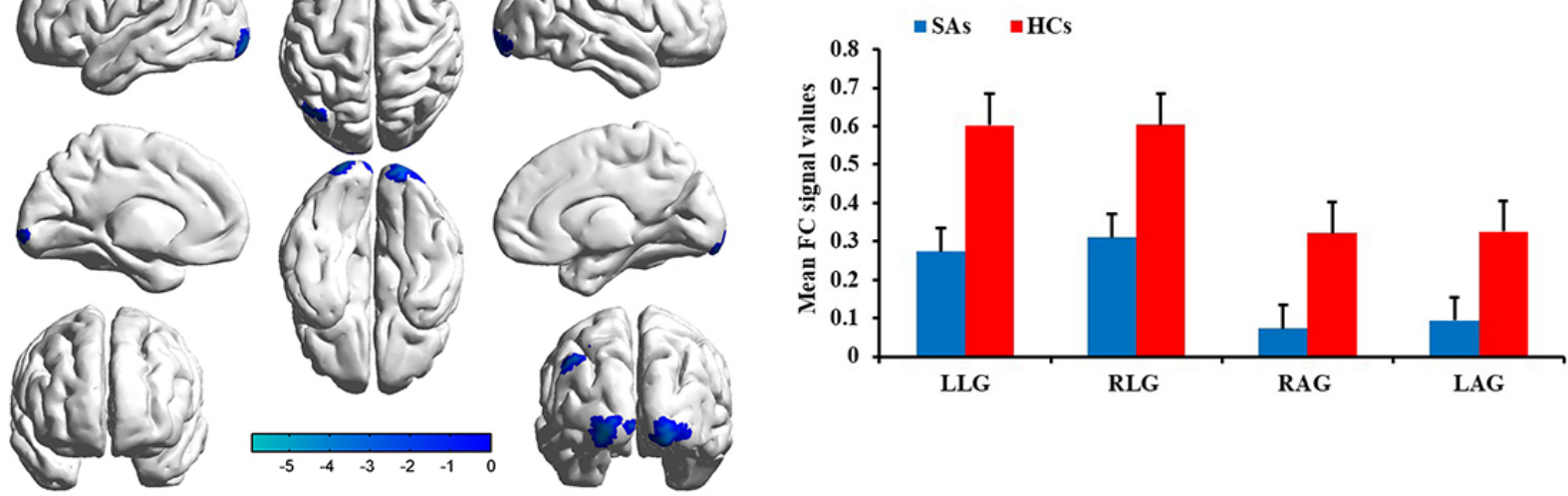

Fig. 1. Brain regions demonstrated statistically significant differences between two groups in FC in the left BA17. Significant FC differences were observed in the left lingual gyrus/right lingual gyrus and left angular gyrus/right angular gyrus, and the blue areas denote lower FC values. SAs, strabismus with amblyopia; HCs, healthy controls; BA, Brodmann area; FC, Functional connectivity.

\section{Methods}

With SA, sixteen adults (5 male and 11 female adults; mean age, $25.67 \pm 4.91$ years) participated at the Department of the Ophthalmology, the First Affiliated Hospital of Nanchang University in Jiangxi Province, China. And 16 HCs (5 males and 11 females; mean age, $24.98 \pm 5.27$ years) were also recruited who were similar in age to the SA group. The common inclusion criteria of the two groups were: (1) adult, over the age of 18 years; (2) availability of MRI scans (with no evidence of a pacemaker or metal implant, etc.). The unique inclusion criteria of the SA group were: (1) diagnosis of strabismus; (2) a difference of more than one line between the bestcorrected visual acuity of amblyopia (VA $>0.20 \log$ MAR units) and the fellow eye; (3) absence of eye trauma, glaucoma, cataract, and other eye diseases. The unique inclusion criteria of the HC group were: (1) normal brain parenchyma on MRI; (2) VA $>1.0$, and the absence of eye disease; (3) absence of mental illness. The common exclusion criteria of the two groups were as follows: (1) the presence of other eye diseases or history of eye surgery; (2) mental illness, cardio- vascular disease, or cerebral infarction; (3) drug or alcohol abuse. The unique exclusion criterion of the SA group was as follows: (1) acquired strabismus.

The age, weight, habitual hand and duration of strabismus amblyopia were collected routinely. The ophthalmologist determined the type of strabismus in adults with SA, measured the strabismus angle, and finally measured the binocular vision of all subjects. The work was approved by the Medical Ethics Committee of the First Affiliated Hospital of Nanchang University and followed the principles of the Declaration of Helsinki. All subjects voluntarily participated in the research and gave informed consent.

\subsection{MRI parameters}

A Trio 3-Tesla MRI scanner (Siemens AG) was used for the MRI scans. Subjects were told to close their eyes but remain awake during the scan of the resting-state fMRI (rsfMRI). The parameters of $\mathrm{T} 1$ and $\mathrm{T} 2$ sequence imaging were as follows: repetition time (TR), $1900 \mathrm{~ms}$; echo time (TE), $2.26 \mathrm{~ms}$; flip angle $(\mathrm{FA}), 9^{\circ}$; matrix, $256 \times 256$; field of view (FOV), $250 \times 250 \mathrm{~mm}$; thickness, $1.0 \mathrm{~mm}$; gap, $0.5 \mathrm{~mm}$; 176 
sagittal slices. A three-dimensional spoiled gradient recalledecho pulse sequence was used to obtain the functional data. The entire scanning procedure lasted approximately $8 \mathrm{~min}$. In addition, 240 structural images were captured using the following settings: acquisition matrix, $64 \times 64$; FOV, $220 \times$ $220 \mathrm{~mm}$; thickness, $4.0 \mathrm{~mm}$; gap, $1.2 \mathrm{~mm}$; TR, $2000 \mathrm{~ms}$; TE, $30 \mathrm{~ms}$; FA, $90^{\circ}$; and 29 axial images.

\section{2 fMRI data processing}

The data processing and analysis process used the Data Processing Assistant for rs-fMRI (DPARSF 2.3, http:// rfmri.org/DPARSF) and the Matlab version 2014b software (Mathworks, Natick, MA, USA), as well as the Statistical Parametric Mapping software (SPM12, The Wellcome Centre for Human Neuroimaging, UCL Queen Square Institute of Neurology, London, UK, http://www.fil.io n.ucl.ac.uk/spm) and the rs-fMRI Data Analysis Toolkit (REST_V1.8_130615, The State key Laboratory of congnitive Neuroscience and learning Beijing Normal University, China, http://www.restfmri.net). The data preprocessing steps were as follows: (1) the first 10 time points were removed to eliminate the interference of slice timing effects. (2) Blood oxygenation level-dependent (BOLD) runs of 240 functional volumes were performed, and the images acquired were motion-corrected and converted to appropriate file formats [20]. The fMRI scans were discarded with an angular displacement or maximum displacement greater than $2 \mathrm{~mm}$. (3) Images were spatially normalized (resampling to $3 \mathrm{~mm}$ isotropic voxels). Images were smoothed with a $6 \mathrm{~mm}$ full width at half maximum Gaussian kernel, linear detrending and temporal band-pass filtering was performed (0.01-0.08 $\mathrm{Hz}$ ). The temporal covariates were later removed, as well as six head-motion parameters, white matter signals, and cerebrospinal fluid signals.

\subsection{Definition of the region of interest in Brodmann area 17}

The V1 is also referred to as Brodmann area 17 (BA17). We chose the WFU PickAtlas2.3 tool (The Functional MRI Laboratory, Wake Forest University School of Medicine, $\mathrm{NC}$, USA) to select each side of V1 as the regions of interest (ROIs) (Table 1).

Table 1. Montreal Neurological Institute coordinates for selected seed regions.

\begin{tabular}{llccc}
\hline ROI & Seed regions & X & Y & Z \\
\hline 1 & L V1 (BA17) & -8 & -76 & 10 \\
2 & R V1 (BA17) & 7 & -76 & 10 \\
\hline
\end{tabular}

ROI, Region of interest.

\subsection{Functional connectivity analysis}

The FC analysis was performed on both the left and right BA17. The Data Processing \& Analysis of Brain Imaging statistical module (http://rfmri.org/dpabi) analyzed the rsfMRI data. The FC graph was constructed using age and sex as covariates. This part of data was analyzed by one-way anal- ysis of covariance and generalized linear model.

\subsection{Statistical analysis}

To evaluate differences in demographic data with SA and HCs, we used the two-sample Student's $t$-test (SPSS software version 20.0, IBM Corp, Armonk, NY, USA). $p<0.05$ was considered statistically significant. To know whether the difference in FC values of different cerebral regions was statistically significant, we used the independent sample $t$-test. The differences in FC between the groups with SA and HCs [at voxel level $p<0.01$ and cluster level $p<0.05$, Gaussian random field-corrected] were determined by applying the twosample $t$-test. In data analysis, we have confirmed cerebral regions with significant differences in $\mathrm{FC}$ values between the two groups. On this basis, we assume that the FC value of the above cerebral regions can be used as a potential indicator for differential diagnosis of the SA group. Finally, we used the receiver operating characteristic (ROC) curve to analyze the potential differential diagnosis value of the above indicators.

\section{Results}

\subsection{Statistical and behavioral changes}

There were no significant differences in age and weight between the SA and HC groups ( $p=0.786$ in age; $p=0.465$ in weight). Besides, there were significant differences in the best-corrected visual acuity between the two groups in the amblyopia eye $(p<0.001)$ and fellow eye $(p=0.035)$. See Table 2 for details.

\subsection{Functional difference}

The values obtained from the $t$-test of the two samples were used to determine the differences in FC mapping between the two groups. The FC values in the left BA17 and left lingual gyrus (BA18), right lingual gyrus (BA18), right angular gyrus (BA39), and left angular gyrus (BA39) were reduced in adults with SA $(\mathrm{n}=18)$ compared with HCs (Fig. 1, Table 3). Moreover, the FC values in the right BA17 and left cuneus (BA18), right inferior occipital gyrus (BA18), and left inferior parietal lobule (BA39) were reduced (Fig. 2, Table 3). We found no significant correlations between the average FC value of specific brain areas in adults with SA and visual acuity $(p>0.05)$.

\subsection{Analysis of receiver operating characteristic curves}

We found significant differences in the FC values of different brain regions between groups with SA and HCs (Fig. 3). The areas under the curve were as follows: (A) left lingual gyrus, 0.910 ( $p<0.001$; 95\% confidence interval [CI], 0.813$1.000)$; right lingual gyrus, 0.883 ( $p<0.001 ; 95 \%$ CI, $0.758-$ $1.000)$; right angular gyrus, $0.813(p=0.003$; 95\% CI, $0.652-$ 0.973); left angular gyrus, 0.844 ( $p=0.001$; 95\% CI, 0.709 0.979) (ROI in left BA17). (B) Left cuneus, 0.914 ( $p<0.001$; 95\% CI, 0.815-1.000); right inferior occipital gyrus, 0.902 ( $p$ $<0.001$; 95\% CI, 0.791-1.000); left inferior parietal lobule, $0.816(p=0.002 ; 95 \% \mathrm{CI}, 0.671-0.962)$ (ROI in right BA17). 

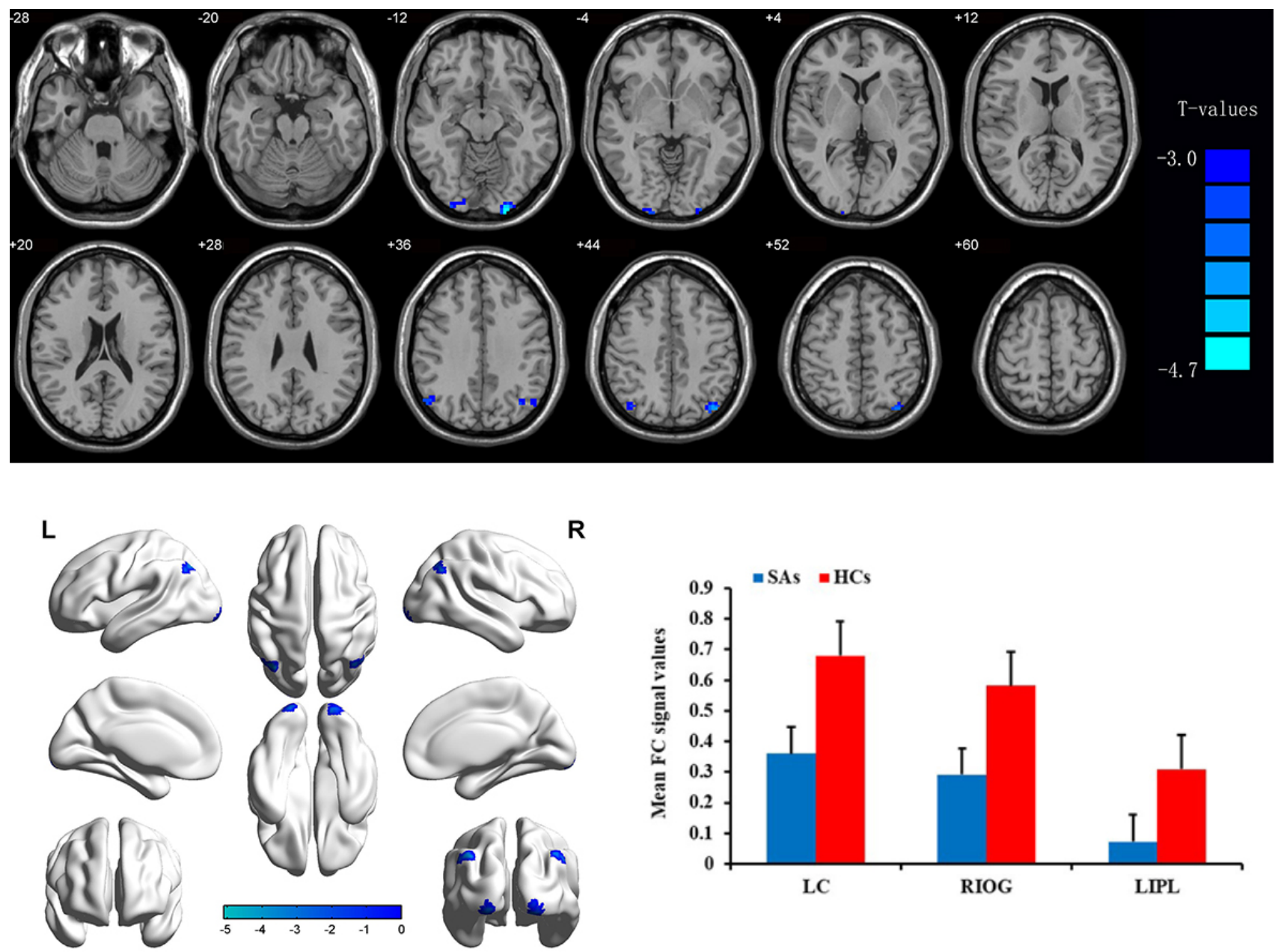

Fig. 2. Brain regions demonstrate statistically significant differences between two groups in FC in the right BA17. Significant FC differences were observed in the left cuneus, right Inferior Occipital Gyrus and left inferior parietal lobule, and the blue areas denote lower FC values. SAs, strabismus with amblyopia; HCs, healthy controls; BA, Brodmann area; FC, Functional connectivity.

Table 2. Demographics and clinical measurements of strabismus with amblyopia and healthy control groups.

\begin{tabular}{lcccc}
\hline Condition & SAs & HCs & $t$ value & $p$-value \\
\hline Male/female & $5 / 11$ & $5 / 11$ & $\mathrm{~N} / \mathrm{A}$ & $>0.99$ \\
Age (years) & $25.67 \pm 4.91$ & $24.98 \pm 5.27$ & -0.243 & 0.786 \\
Weight (kg) & $61.22 \pm 3.67$ & $59.18 \pm 3.65$ & -0.716 & 0.465 \\
Handedness & $16 \mathrm{R}$ & $16 \mathrm{R}$ & $\mathrm{N} / \mathrm{A}$ & $>0.99$ \\
Duration (years) & $23.57 \pm 4.91$ & $\mathrm{~N} / \mathrm{A}$ & $\mathrm{N} / \mathrm{A}$ & $\mathrm{N} / \mathrm{A}$ \\
Esotropia/exotropia & $6 / 10$ & $\mathrm{~N} / \mathrm{A}$ & $\mathrm{N} / \mathrm{A}$ & $\mathrm{N} / \mathrm{A}$ \\
Angle of strabismus (PD) & $31.15 \pm 11.65$ & $\mathrm{~N} / \mathrm{A}$ & $\mathrm{N} / \mathrm{A}$ & $\mathrm{N} / \mathrm{A}$ \\
VA-AE & $0.15 \pm 0.10$ & $1.05 \pm 0.25$ & 6.136 & $<0.001$ \\
VA-FE & $0.55 \pm 0.05$ & $1.00 \pm 0.10$ & 1.151 & 0.035 \\
\hline
\end{tabular}

Independent $t$-tests comparing the two groups $(p<0.05$ represented statistically significant differences). Data are shown as mean \pm standard deviation or not applicable. SA, strabismus with amblyopia; HC, healthy control; R, right; PD, prism diopter; AE, amblyopic eye; FE, fellow eye; VA, Visual acuity.

\section{Discussion}

Adults with other eye diseases reportedly exhibit specific changes in FC of the V1 and other areas [21-32]. There was still a lack of understanding of the changes in cerebral func- tion in adults with strabismus amblyopia. We found reduced FC values between the left BA17 and bilateral lingual/angular gyri in adults with SA compared with HCs. Similarly, the FC values between the right BA17 and left cuneus, right inferior occipital gyrus and left inferior parietal lobule are all reduced. 
A

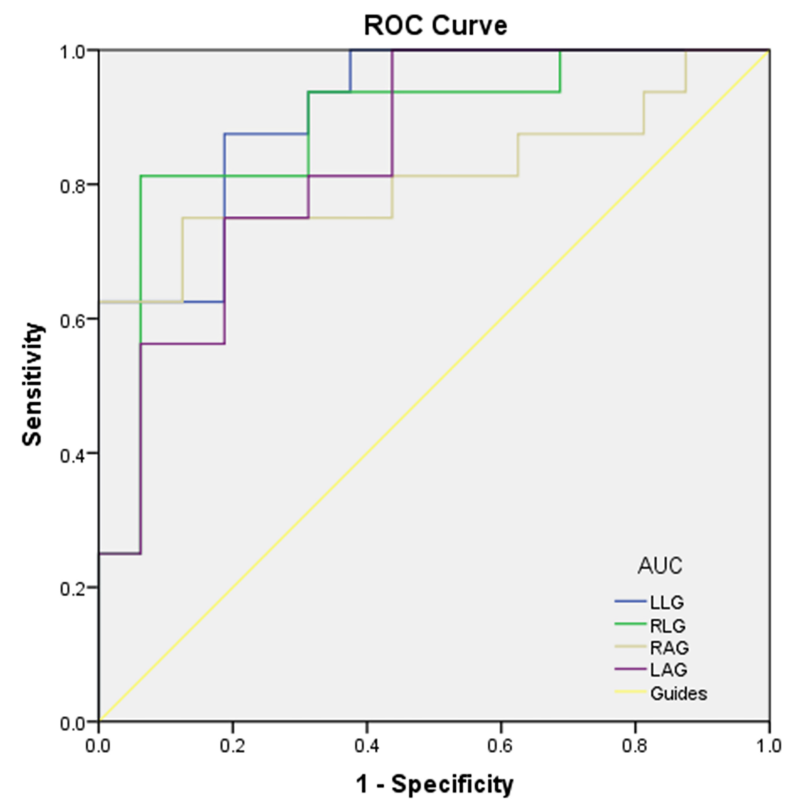

B

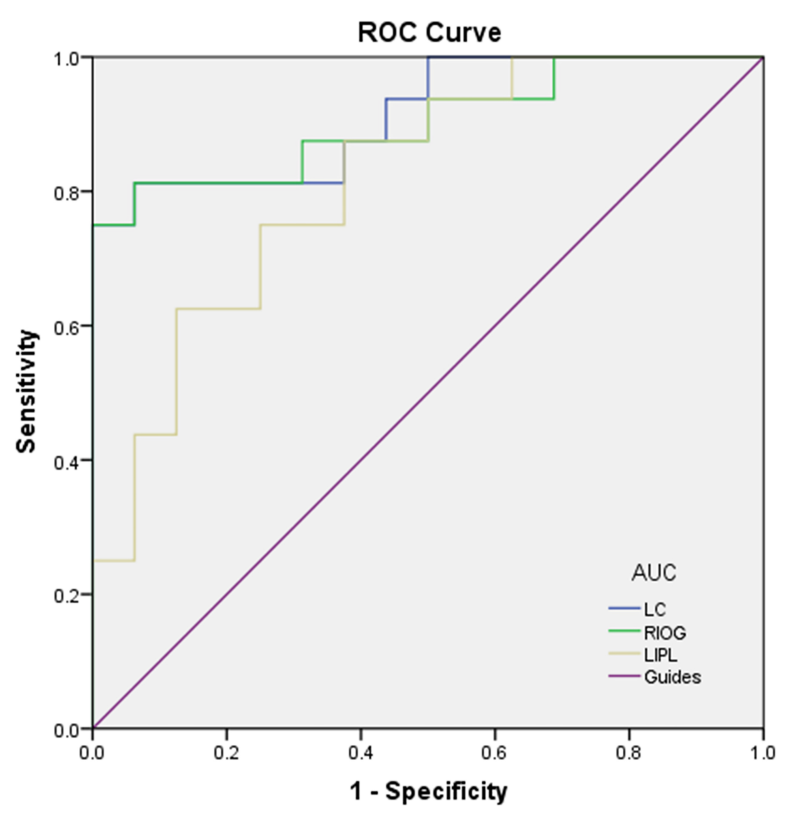

Fig. 3. ROC curve analysis of the mean FC values for altered brain regions. (A) ROI in left BA17. The AUCs of different brain regions were as follows: left lingual gyrus, 0.910 ( $p<0.001$; 95\% confidence interval [CI], 0.813-1.000); right lingual gyrus, 0.883 ( $p<0.001 ; 95 \%$ CI, 0.758-1.000); right angular gyrus, 0.813 ( $p=0.003$; 95\% CI, 0.652-0.973); left angular gyrus, 0.844 ( $p=0.001$; 95\% CI, 0.709-0.979). (B) ROI in right BA17. The AUCs of different brain regions were as follows: Left cuneus, 0.914 ( $p<0.001$; 95\% CI, 0.815-1.000); right inferior occipital gyrus, 0.902 ( $p<0.001$; 95\% CI, 0.791-1.000); left inferior parietal lobule, 0.816 ( $s=0.002$; 95\% CI, 0.671-0.962). ROC, receiver operating characteristic; FC, Functional connectivity; CI, Confidence interval; SAs, strabismus with amblyopia; HCs, healthy controls; LC, left cuneus; RIOG, right Inferior occipital gyrus; LIPL, left inferior parietal lobule; LLG, left lingual gyrus; RLG, right lingual gyrus; RAG, right angular gyrus; LAG, left angular gyrus.

Table 3. Brain regions with significant differences in FC between SAs and HCs.

\begin{tabular}{|c|c|c|c|c|c|c|c|c|}
\hline \multirow{7}{*}{ FC-L } & \multirow{2}{*}{ Brain areas } & \multicolumn{3}{|c|}{ MNI coordinates } & \multirow{2}{*}{ BA } & \multirow{2}{*}{ Peak voxels } & \multirow{2}{*}{$t$ value } & \multirow{2}{*}{$p$-value } \\
\hline & & $X$ & $\mathrm{Y}$ & $\mathrm{Z}$ & & & & \\
\hline & \multicolumn{8}{|l|}{ ROI in left BA17 } \\
\hline & L Lingual Gyrus & -24 & -102 & -12 & 18 & 42 & -5.1051 & $p<0.005$ \\
\hline & R Lingual Gyrus & 24 & -102 & -6 & 18 & 37 & -4.1837 & $p<0.005$ \\
\hline & R Angular Gyrus & 54 & -66 & 39 & 39 & 32 & -3.8005 & $p<0.005$ \\
\hline & L Angular Gyrus & -39 & -75 & 48 & 39 & 83 & -4.169 & $p<0.005$ \\
\hline \multirow{6}{*}{ FC-R } & Brain areas & \multicolumn{3}{|c|}{ MNI coordinates } & \multirow{2}{*}{$\mathrm{BA}$} & \multirow{2}{*}{ Peak voxels } & \multirow{2}{*}{$t$ value } & \multirow{2}{*}{$p$-value } \\
\hline & & $\mathrm{X}$ & $\mathrm{Y}$ & $\mathrm{Z}$ & & & & \\
\hline & ROI in right BA17 & & & & & & & \\
\hline & L Cuneus & -24 & -102 & -12 & 18 & 106 & -5.9432 & $p<0.005$ \\
\hline & R Inferior Occipital Gyrus & 24 & -102 & -6 & 18 & 84 & -4.9365 & $p<0.005$ \\
\hline & L Inferior Parietal Lobule & -48 & -72 & 42 & 39 & 42 & -3.6918 & $p<0.005$ \\
\hline
\end{tabular}

The statistical threshold was set at voxel level with $p<0.005$ for multiple comparisons using Gaussian random field theory cluster level with $p<0.05$ and cluster size $>40$ voxels, AlphaSim corrected. $t$ value was the statistical result obtained by the independent sample $t$ test carried out by the Gaussian random field theory. FC, Functional Connectivity; SA, strabismus with amblyopia; HC, healthy control; L, left; R, right; MNI, Montreal Neurological Institute; BA, Brodmann area.

The lingual gyrus is connected to the fusiform gyrus and the parahippocampal gyrus and processed complex visual stimuli by identifying basic characteristics [21]. The lingual gyrus is also associated with processing overall visual stimuli.
When features such as visual contrast are increased, activation of the lingual gyrus is decreased [22].

The functional connection between the left BA17 and the bilateral lingual gyrus decreased. The lingual gyrus partici- 
pated in several vision-related functional activities, so the reduced connectivity of functional activity in this part of the cerebrum can be seen to be a potential cause of visual fusion disorders in adults with SA. The angular gyrus activity is positively correlated with a delay in visual, auditory, and audiovisual actions; thus, differences between the predicted and actual results may be detected [23]. Block et al. [24] found that a specific region in the angular gyrus plays a positive mediating role between visual proprioception and rearrangement.

Ho et al. [25, 26] also found that the angular gyrus plays a key role in the lateralization of visual-spatial attention. Near and far spatial vision is reportedly altered in the right supramarginal gyrus under transcranial magnetic stimulation (TMS). In contrast, only "near space" vision is altered in the right angular gyrus under TMS [27]. These results indicate that the angular gyrus plays an important role in near-spatial visual processing and spatial attention.

The functional connection between left BA17 and bilateral angular gyrus is significantly reduced in adults with SA. The angular gyrus plays an important role in spatial visual processing, spatial attention, visual proprioception and audiovisual activities. Therefore, the decrease of functional connections between the angular gyrus and other cerebral regions could be one of the important pathological mechanisms of visual function decline in the SA group. Of course, the decrease of FC in this particular cerebral area may also result from visual dysfunction caused by strabismus amblyopia, which leads to significant changes in the functional activity of the cerebral cortex. Visual stimuli activated the primary posterior visual area and the anteromedial cuneus from the beginning of the cortical response. The anteromedial cuneus is in an appropriate temporal position to interact with the V1, thereby modifying the information transmitted by $\mathrm{V} 1$ to the outer cortex [28].

In addition, through the fMRI analysis of the amplitude of low-frequency fluctuation (ALFF) sequence, some scholars have pointed out that the ALFF value of the precuneus of adults with persistent postural-perceptual vertigo is significantly lower. We can infer that the precuneus played an important role in visual and vestibular information processing. We found that the FC value of the left cuneus of the SA group decreased significantly. The decreased functional connectivity of this cerebral region may be one of the important pathological mechanisms of visual fusion and vestibular dysfunction [29].

The FC values were reduced in the right BA17 and left cuneus group with SA, which may explain their visual deficits. Lu et al. [30] suggested that morphological abnormalities in the occipital, temporal, and frontal cortices of adults with amblyopia were due to early abnormal visual experience, which may cause neurodevelopmental processes to interfere with brain maturation. These changes may affect visual acuity and binocular vision.

Sato et al. [31] indicated that the degree of activation of the right inferior occipital gyrus increases, regardless of whether the eyes are looking at a dynamic or a static pattern. This suggests that the right inferior occipital gyrus is early in visual processing. The results found that the functional connection between the right BA17 and the right inferior occipital gyrus was significantly decreased in adults with SA. The above discussion and analysis showed that the right inferior occipital gyrus played an important role in various visual modes. Therefore, a significant decrease in the functional connectivity of the right inferior occipital gyrus could be one of the potential auxiliary indicators for the differential diagnosis of SA.

The dorsal visual pathway starts from V1 and reaches the inferior parietal lobe through V2 and the medial temporal area, where the visual information is used to analyze and guide eye movement [32]. Yan et al. [33] have found that the structure of the inferior parietal lobe of patients with concomitant exotropia is abnormal. In addition, Ding et al. [34] indicated that the decreased FC values between the primary visual area and the inferior parietal lobule in a group with amblyopia might reflect functional deficits in the dorsal stream. Similarly, adults with SA had abnormal functional connections between the right BA17 and left inferior parietal lobe, leading to visual deficits.

Furthermore, we found the FC between the primary visual cortex and the medial/lateral occipitotemporal gyrus in SAs was not abnormal compared with healthy subjects. However, many scholars think the medial/lateral occipitotemporal gyrus in the role of vision cannot be ignored.

We further speculate that strabismus amblyopia can lead to significant changes in functional connection activity in specific brain regions. This change may be one of the potential neuropathological mechanisms of strabismus amblyopia. Of course, this inference requires further research. Through our above analysis and discussion, we have known that the occurrence and development of strabismus amblyopia are closely related to the complex brain network, especially the oculomotor neural network in the primary visual cortex [35]. The changes in neurological activity in the above brain areas may result from SA and the compensatory changes of cerebral cortex function caused by SA or its specific pathological mechanism. The changes in functional activities in the above brain regions may result from visual dysfunction caused by SA. They may be one of the potential neuropathological mechanisms of SA.

\section{Conclusions}

We can conclude that the functional connections of specific cerebral regions in adults with SA were significantly altered compared with those in normal adults. These include bilateral lingual gyrus, bilateral angular gyrus, left cuneus gyrus, right inferior occipital gyrus, and left inferior parietal lobule. The functional connection changes of the above cerebral regions may be the potential pathological mechanism of the occurrence and development of SA. The specific pathological mechanism of strabismus amblyopia needs to be fur- 
ther studied. In addition, in clinical diagnosis, the above signal values of abnormal functional connectivity in brain areas may be used as indicators for the auxiliary diagnosis of SA diseases.

\section{Abbreviations}

ROI, region of interest; SA, strabismus with amblyopia; $\mathrm{HC}$, healthy control; $\mathrm{R}$, right; $\mathrm{PD}$, prism diopter; $\mathrm{AE}$, amblyopic eye; FE, fellow eye; VA, Visual acuity; FC, functional connectivity; MNI, Montreal Neurological Institute; BA, Brodmann area; ROC, receiver operating characteristic; CI, Confidence interval; LC, left cuneus; RIOG, right Inferior occipital gyrus; LIPL, left inferior parietal lobule; LLG, left lingual gyrus; RLG, right lingual gyrus; RAG, right angular gyrus; LAG, left angular gyrus.

\section{Author contributions}

LQL, QYL, ZHZ and YS conceived and designed the experiments; SNW and HYS performed the experiments; LJZ and RBL analyzed the data; YCP contributed reagents and materials; LQL and ZHZ wrote the paper.

\section{Ethics approval and consent to participate}

The study methods and protocols were approved by the Medical Ethics Committee of the First Affiliated Hospital of Nanchang University (Nanchang, China) (2015016) and followed the principles of the Declaration of Helsinki. All subjects were notified of the objectives and content of the study and latent risks, and then provided written informed consent to participate.

\section{Acknowledgment}

We thank two/three anonymous reviewers for excellent criticism of the article.

\section{Funding}

National Natural Science Foundation (No: 82160195); Central Government Guides Local Science and Technology Development Foundation (No: 20211ZDG02003); Key Research Foundation of Jiangxi Province (No: 20181BBG70004, 20203BBG73059); Excellent Talents Development Project of Jiangxi Province (No: 20192BCBL23020); Natural Science Foundation of Jiangxi Province (No: 20181BAB205034); Grassroots Health Appropriate Technology “Spark Promotion Plan" Project of Jiangxi Province (No: 20188003); Health Development Planning Commission Science Foundation of Jiangxi Province (No: 20201032, 202130210).

\section{Conflict of interest}

The authors declare no conflict of interest.

\section{References}

[1] Chen X, Fu Z, Yu J, Ding H, Bai J, Chen J, et al. Prevalence of amblyopia and strabismus in Eastern China: results from screening of preschool children aged 36-72 months. British Journal of Ophthalmology. 2016; 100: 515-519.
[2] Zhang W. Interpretation the consensus of strabismus classification. Chinese Journal of Ophthalmology. 2015; 51: 406-407. (In Chinese)

[3] Yang XB, Liu LQ. Research progress on visual defects of amblyopia. Chinese Journal of Experimental Ophthalmology. 2017; 35: 1139-1142. (In Chinese)

[4] Shapira Y, Machluf Y, Mimouni M, Chaiter Y, Mezer E. Amblyopia and strabismus: trends in prevalence and risk factors among young adults in Israel. The British journal of ophthalmology. 2018; 102: 659-666.

[5] Pan C, Chen X, Zhu H, Fu Z, Zhong H, Li J, et al. School-based assessment of amblyopia and strabismus among multiethnic children in rural China. Scientific Reports. 2017; 7: 13410.

[6] Elflein HM, Krause L, Rommel A, Urschitz MS, Pfeiffer N, Schuster AK. Do children with strabismus receive ophthalmic treatment? Results from the KiGGS (German Health Interview and Examination Survey for Children and Adolescents) baseline survey (2003-2006). Ophthalmology. 2019; 116: 1194-1199. (In German)

[7] Sabri K, Knapp CM, Thompson JR, Gottlob I. The VF-14 and Psychological Impact of Amblyopia and Strabismus. Investigative Opthalmology \& Visual Science. 2006; 47: 4386-4392.

[8] Koklanis K, Abel LA, Aroni R. Psychosocial impact of amblyopia and its treatment: a multidisciplinary study. Clinical and Experiment Ophthalmology. 2006; 34: 743-750.

[9] Deng JJ, Qiang J, He XG. Research Progress in Understanding the Relationship Treatment of Strabismus, Amblyopia and the Mental Health of Children. Chinese Journal of Optometry Ophthalmology and Visual Science. 2017; 19: 694-698. (In Chinese)

[10] Yan XH, Wang Y, Xu LJ, Liu Y, Song S, Ding K, et al. Altered Functional Connectivity of the Primary Visual Cortex in Adult Comitant Strabismus: A Resting-State Functional MRI Study. Current Eye Research. 2019; 44: 316-323.

[11] Thompson B, Maehara G, Goddard E, Farivar R, Mansouri B, Hess RF. Long-Range Interocular Suppression in Adults with Strabismic Amblyopia: A Pilot fMRI Study. Vision. 2019; 3: 2.

[12] Jia CH, Lu GM, Zhang ZQ, Wang Z, Huang W, Ma F, et al. Comparison of deficits in visual cortex between anisometropic and strabismic amblyopia by fMRI retinotopic mapping. Zhonghua Yi Xue Za Zhi. 2010; 90: 1446-1452. (In Chinese)

[13] Li QH, Zhu PW, Shao Y. Research progresses of brain remodeling in visual deprivation based on fMRI. Chinese Journal of Medical Imaging Technology. 2018; 34: 1893-1896. (In Chinese)

[14] Cox MA, Dougherty K, Westerberg JA, Schall MS, Maier A. Temporal dynamics of binocular integration in primary visual cortex. Journal of Vision. 2019; 19: 13.

[15] Read JC, Cumming BG. Testing Quantitative Models of Binocular Disparity Selectivity in Primary Visual Cortex. Journal of Neurophysiology. 2003; 90: 2795-2817.

[16] Zhang NY, Zhu XH, Yacoub E. Functional MRI mapping neuronal inhibition and excitation at columnar level in human visual cortex. Experimental Brain Research. 2010; 204: 515-524.

[17] Chen Z, Zhou Q, Calhoun V. Phase fMRI informs whole-brain function connectivity balance across lifespan with connectionspecific aging effects during the resting state. Brain Structure and Function. 2019; 224: 1489-1503.

[18] Muckli L. What Are We Missing Here? Brain Imaging Evidence for Higher Cognitive Functions in PrimaryVisual Cortex V1. International Journal of Imaging Systems and Technology. 2010; 20: $131-139$.

[19] Bulte D. Hyperoxia and Functional MRI. Advances in Experimental Medicine and Biology. 2016; 903: 187-199.

[20] Bandettini PA, Jesmanowicz A, Wong EC. Processing strategies for time-course data sets in functional MRI of the human brain. Magnetic Resonance in Medical Sciences. 1993; 30: 161-173.

[21] Stanislav AK Maria MP, Anna VL Alexander VV, Andrey AK. Activation of left lingual gyrus related to working memory for 
schematic faces. International Journal of Psychophysiology. 2014; 94: 241.

[22] Mechelli A, Humphreys GW, Mayall K, Olson A, Price CJ. Differential effects of word length and visual contrast in the fusiform and lingual gyri during reading. Proceedings of The Royal Society B-Biological Sciences. 2000; 267: 1909-1913.

[23] van Kemenade BM, Arikan BE, Kircher T, Straube B. The angular gyrus is a supramodal comparator area in action-outcome monitoring. Brain Structure and Function. 2017; 222: 3691-3703.

[24] Block H, Bastian A, Celnik P. Virtual Lesion of Angular Gyrus Disrupts the Relationship between Visuoproprioceptive Weighting and Realignment. Journal of Cognitive Neuroscience. 2013; 25: 636-648.

[25] Ho AK, Nestor PJ, Williams GB, Bradshaw JL, Sahakian BJ, Robbins TW, et al. Pseudo-neglect in Huntington's disease correlates with decreased angular gyrus density. NeuroReport. 2004; 15: 1061-1064.

[26] Cattaneo Z, Silvanto J, Pascual-Leone A, Battelli L. The role of the angular gyrus in the modulation of visuospatial attention by the mental number line. NeuroImage. 2009; 44: 563-568.

[27] Bjoertomt O, Cowey A, Walsh V. Near space functioning of the human angular and supramarginal gyri. Journal of Neuropsychology. 2009; 3: 31-43.

[28] Vanni S, Tanskanen T, Seppa M, Uutela K, Hari R. Coinciding early activation of the human primary visual cortex and antero- medial cuneus. Proceedings of the National Academy of Sciences. 2001; 98: 2776-2780.

[29] Li K, Si L, Cui B, Ling X, Shen B, Yang X. Altered spontaneous functional activity of the right precuneus and cuneus in patients with persistent postural-perceptual dizziness. Brain Imaging and Behavior. 2020; 14: 2176-2186.

[30] Lu L, Li Q, Zhang L, Tang S, Yang X, Liu L, et al. Altered cortical morphology of visual cortex in adults with monocular amblyopia. Journal of Magnetic Resonance Imaging. 2019; 50: 1405-1412.

[31] Sato W, Kochiyama T, Uono S, Matsuda K, Usui K, Usui N, et al. Rapid gamma oscillations in the inferior occipital gyrus in response to eyes. Scientific Reports. 2016; 6: 36321.

[32] de la Malla C, Brenner E, de Haan, Edward HF. A visual illusion that influences perception and action through the dorsal pathway. Communications Biology. 2019; 2: 38.

[33] Yan XH, Lin XM, Wang QF, Zhang YC, Chen YM, Song SJ, et al. Dorsal Visual Pathway Changes in Patients with Comitant Extropia. PLoS ONE. 2010; 5: e10931.

[34] Ding K, Liu Y, Yan X, Lin X, Jiang T. Altered Functional Connectivity of the Primary Visual Cortex in Subjects with Amblyopia. Neural Plasticity. 2013; 2013: 1-8.

[35] Yan X, Wang Y, Xu L, Liu Y, Song S, Ding K, et al. Altered Functional Connectivity of the Primary Visual Cortex in Adult Comitant Strabismus: A Resting-State Functional MRI Study. Current Eye Research. 2019; 44: 316-323. 